\title{
Kalman estimator- and general linear model-based on-line brain activation mapping by near-infrared spectroscopy
}

\author{
Xiao-Su Hu${ }^{1}$, Keum-Shik Hong ${ }^{1,2^{*}}$, Shuzhi S Ge ${ }^{1,3}$, Myung-Yung Jeong ${ }^{1}$
}

\author{
* Correspondence: kshong@pusan. \\ ac.kr \\ ${ }^{1}$ Department of Cogno- \\ Mechatronics Engineering, Pusan \\ National University; 30 Jangjeon- \\ dong, Geumjeong-gu, Busan609- \\ 735, Korea
}

\begin{abstract}
Background: Near-infrared spectroscopy (NIRS) is a non-invasive neuroimaging technique that recently has been developed to measure the changes of cerebral blood oxygenation associated with brain activities. To date, for functional brain mapping applications, there is no standard on-line method for analysing NIRS data.

Methods: In this paper, a novel on-line NIRS data analysis framework taking advantages of both the general linear model (GLM) and the Kalman estimator is devised. The Kalman estimator is used to update the GLM coefficients recursively, and one critical coefficient regarding brain activities is then passed to a t-statistical test. The $t$-statistical test result is used to update a topographic brain activation map. Meanwhile, a set of high-pass filters is plugged into the GLM to prevent very lowfrequency noises, and an autoregressive (AR) model is used to prevent the temporal correlation caused by physiological noises in NIRS time series. A set of data recorded in finger tapping experiments is studied using the proposed framework.

Results: The obtained results suggest that the method can effectively track the task related brain activation areas, and prevent the noise distortion in the estimation while the experiment is running. Thereby, the potential of the proposed method for real-time NIRS-based brain imaging was demonstrated.

Conclusions: This paper presents a novel on-line approach for analysing NIRS data for functional brain mapping applications. This approach demonstrates the potential of a real-time-updating topographic brain activation map.
\end{abstract}

\section{Background}

Near-infrared spectroscopy (NIRS), an emerging brain imaging technique, measures the hemodynamic changes that effectively reflect the brain activity occurring while people perform a wide range of mental tasks [1-5]. It can provide both topographic $[2,4,6]$ and tomographic [1,7] brain images. Specifically, NIRS monitors the regional cerebral blood flow (rCBF) variation by measuring, through the skull, the absorption changes of nearinfrared light at wavelengths between $650 \mathrm{~nm}$ and $950 \mathrm{~nm}$ [3]. These changes are caused by the concentration variations of oxy-hemoglobin $(\mathrm{HbO})$ and deoxy-hemoglobin $(\mathrm{HbR})$, two primary absorbing chromophores in brain capillary blood.

NIRS, compared with other prevalent brain imaging and activity measurement techniques such as electroencephalography (EEG) and functional magnetic resonance imaging (fMRI), offers itself as a trade-off between spatial and temporal resolutions. The

(C) $2010 \mathrm{Hu}$ et al; licensee BioMed Central Ltd. This is an Open Access article distributed under the terms of the Creative Commons Attribution License (http://creativecommons.org/licenses/by/2.0), which permits unrestricted use, distribution, and reproduction in any medium, provided the original work is properly cited. 
usability and drawbacks of NIRS methods, as discussed in a detailed review and comparison with other neuroimaging methods, was provided by Perrey [6]. fMRI has been used over the past decade in a growing number of applications. The critical drawbacks of the fMRI-based approaches, however, are the cost and the non-portability of the fMRI scanner. In fact, another comprehensive review [8], in comparing the respective features of NIRS and fMRI, concluded that NIRS has great potentials for neurological and psychiatric applications, due to its simplicity, portability, and insensitivity to motion artifacts. Meanwhile, the EEG technique is limited, due to its poor spatial resolution and low signal-to-noise ratios in many applications; NIRS can provide comparatively better quality in these aspects [9]. Indeed NIRS, in its wide applicability, might help to bring functional imaging to the patient's bedside [3].

\section{Methods}

There is currently no standard method of topographic NIRS data analysis for brain mapping. In NIRS detection of hemodynamic responses, the light attenuation measured by the equipment needs to be converted to $\mathrm{HbO}$ and HbR concentration changes via the modified Beer-Lambert law (MBLL) [10]. Hence, a differential path length factor (DPF, intra- and inter-subject varying) in the MBLL has to be assumed to account for the increase of the path length between a source and a detector [11].

The classical approach in topographic NIRS data analysis is a paired $t$-test to determine if a concentration change between two states (for instance, "rest" vs. "task") is statistically significant. Many researchers nowadays use this approach [12-14], because it is simple and, thus, can provide a quick assessment to the task. One of the most popular tools in this regard is a Matlab-based program known as HomER ([15] http:// www.nmr.mgh.harvard.edu/PMI/).

However, there are limitations to the classical $t$-test. First, a maximum activation period needs to be predefined. The remaining temporal information not included in the defined activation period therefore is ignored, leading to underestimation of brain activation. Another problem is the DPF assumption. Since the DPF is intra- and intersubject variant and impossible to be measured for every measurement location with commonly obtainable continuous wave NIRS equipment, use of a constant DPF leads to biased estimation of concentration changes [16].

To overcome such problems of the classical $t$-test, a number of research groups have used various general linear model (GLM)-based methods for analysis of NIRS data [16-20]. The GLM-based methods were initially developed for fMRI-based functional brain mapping [21]. The GLM is a statistical linear concept that explains measured data in the form of a linear combination of several explanatory variables plus an error term. The explanatory variables, modelled according to the time course, separately account for the brain-activity-evoked signals and noises. Therefore, the estimation of brain activity is reduced to the problem of estimating the relevant coefficients with proper statistics.

The GLM-based methods negate the need for user-defined rest and task periods, because the response is modelled according to the entire time course. The temporal information over the entire time course, thus, is examined. On the other hand, the GLM-based methods investigate the temporal variation pattern of the signal, and estimate the coefficients with statistics at different measurement locations, separately. Therefore, these methods are robust in cases where an assumed constant DPF is used. 
GLM-based methods, however, cannot offer on-line analysis. This constrains their use in applications where real-time information or feedback is required. Real-time brain imaging data analysis in comparison with off-line methods, significantly, improves the information acquisition rate and the feedback speed. Furthermore, it may potentially benefit the development of brain-computer interfaces $(\mathrm{BCI})$.

The critical task in modifying a GLM-based method on-line is the recursive estimation of GLM coefficients. Previous researchers have used different methods to achieve recursive estimation, including recursive least square [22], Cholesky-decompositionbased recursive least square [23], and Kalman filtering [24,25]. All these approaches can effectively and recursively estimate GLM coefficients.

It is not sufficient to draw an updated brain activation map only by estimating GLM coefficients on-line. It is known that NIRS time series contain noises from different sources. Very-low-frequency noises caused by optodes shifts or slow cardiac/vascular artifacts [26], for example, might lead to biased estimation. The temporal correlation caused by physiological (cardiac, respiratory, blood pressure) noises might lead to an inflated $t$-value and, thereby, overestimation of brain activation. Furthermore, for functional brain mapping applications, a statistical test is very important, since it will provide significance verification of the derived estimation. All these issues with regard to on-line versions of GLM-based methods need to be addressed.

On-line estimation of GLM coefficients is generally discussed in [24]. In [25], the whole framework provided on-line estimation of GLM coefficients, and a relevant statistical test analysed the fMRI data. However, this work did not consider temporal correlation in fMRI data, which might also exist in NIRS data. In [26], a framework for NIRS-based BCI applications was developed that can estimate GLM coefficients without statistical information in classifying different hand tasks in real-time. In [27], the feasibility of estimating GLM coefficients on-line using a Kalman filter by studying a set of fMRI data was examined.

In the present study, we develop an on-line Kalman-estimator- and GLM-based NIRS data processing framework for brain activation mapping. We aim to answer two questions. (i) Is it possible to covert the off-line GLM-based method to an online version for NIRS-based brain activation mapping? The proposed method can provide updated brain activation maps on-line as well as track task-related brainactive areas from an early stage while the experiment is running. (ii) Can the proposed method prevent noises that distort the estimation while data is sequentially incorporated?

\section{NIRS measurement system and experimental procedure}

Five right-handed healthy volunteers (all male, aged 24 to 34 years) participated in the experiment. None of the participants had a history of any neurological disorder. All of the participants provided written informed consent. The experiment was conducted in accordance with the latest Declaration of Helsinki. The data were acquired with a continuous-wave NIRS imaging system (DYNOT: DYnamic Near-infrared Optical Tomography) obtained from NIRx Medical Technologies, Brooklyn, NY, at a sampling rate of $1.81 \mathrm{~Hz}$. The system emits laser lights of different wavelengths $(760 \mathrm{~nm}$ and $830 \mathrm{~nm}$ ) from each source. Figure 1 shows the channel distribution and measurement location. The distance between different optodes is $2 \mathrm{~mm}$. 


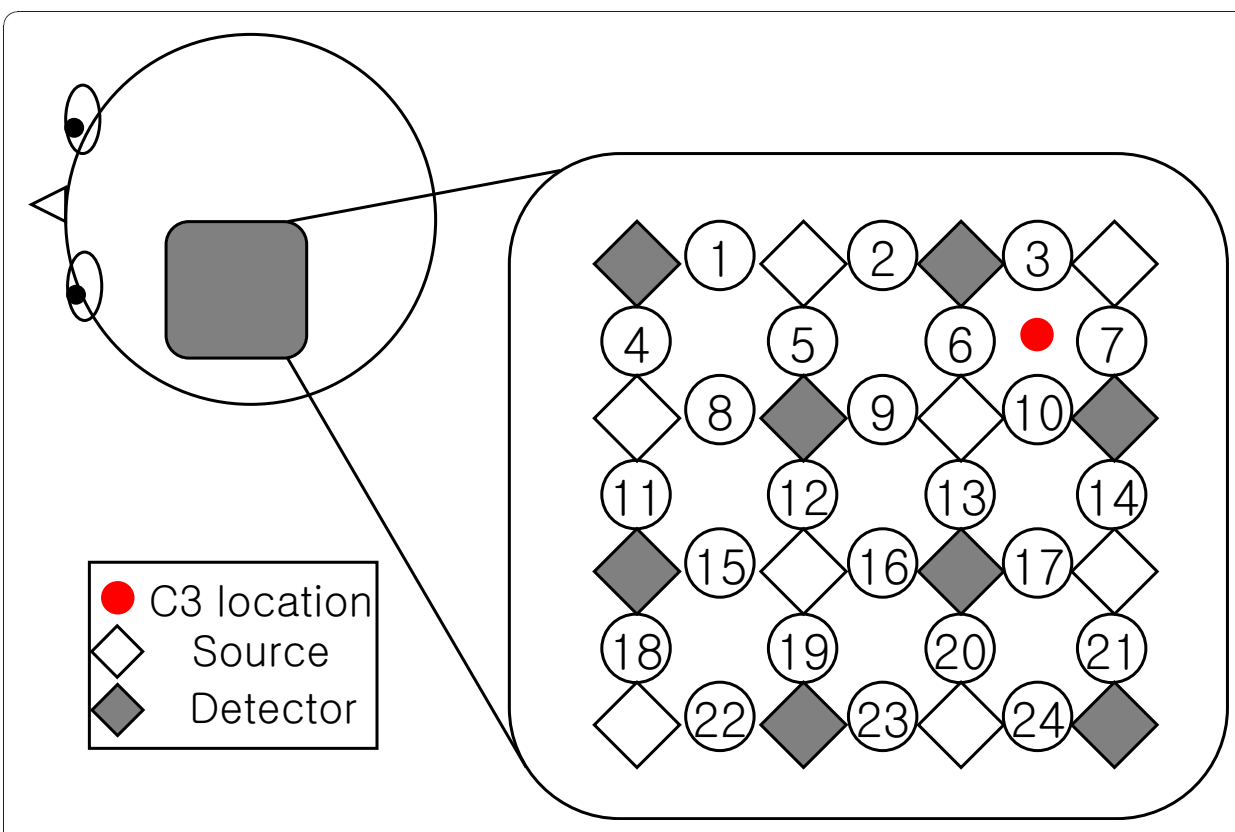

Figure 1 Channel distribution and measurement location on head. The detected area covers the primary motor cortex, dorsolateral prefrontal cortex, and Broca's area of the left hemisphere of the subjects. The C3 location, in the international 10-20 system, is used as a reference.

In the experiment, the subjects were asked to perform a finger-tapping task. The experiment consisted of a $42 \mathrm{sec}$ preparation period and 10 sessions. Each session included a $21 \mathrm{sec}$ finger-tapping period and a $30 \mathrm{sec}$ rest period. Accordingly, the total duration of the experiment was $552 \mathrm{sec}$.

\section{Analysis framework of NIRS data}

A schematic summarization of our method is as follows. (i) The relative concentration changes of two blood chromospheres, $\mathrm{HbO}$ and $\mathrm{HbR}$, are calculated via MBLL [10,27]; (ii) A linear model is built according to the experimental procedure to fit the relative $\mathrm{HbO}$ concentration change. The model describes both the signals corresponding to the brain activity and the noise; (iii) The model coefficients are recursively estimated using the Kalman estimator; (iv) At every time step, the brain-activity-related coefficient is selected, and then passed to the $t$-statistical test to determine if its value is statistically greater than zero (a value greater than zero indicates brain activity): In this way, a probability brain activation map is drawn. Figure 2 is a schematic flow chart of the framework.

\section{NIRS measurement model}

In NIRS measurement, the optical density variation $(\triangle O D)$ can be expressed as a linear combination of hemoglobin concentration changes $\left(\Delta C_{H b O}\right.$ and $\left.\Delta C_{H b R}\right)$ multiplied by proper coefficients. Their relationship is described in MBLL terms as

$$
\Delta O D^{\lambda, i}=\alpha^{\lambda} \Delta C L^{\lambda, i} D P F^{\lambda}
$$

where $a^{1} \Delta C=a_{H b O}^{1} \Delta C_{H b O}+a_{H b R}^{1} \Delta C_{H b R}, \lambda$ is the wavelength of the laser source, $i$ indicates channel number, $\alpha_{H b O}\left[\mu \mathrm{M}^{-1} \mathrm{~mm}^{-1}\right]$ and $\alpha_{H b R}\left[\mu \mathrm{M}^{-1} \mathrm{~mm}^{-1}\right]$ are the extinction 


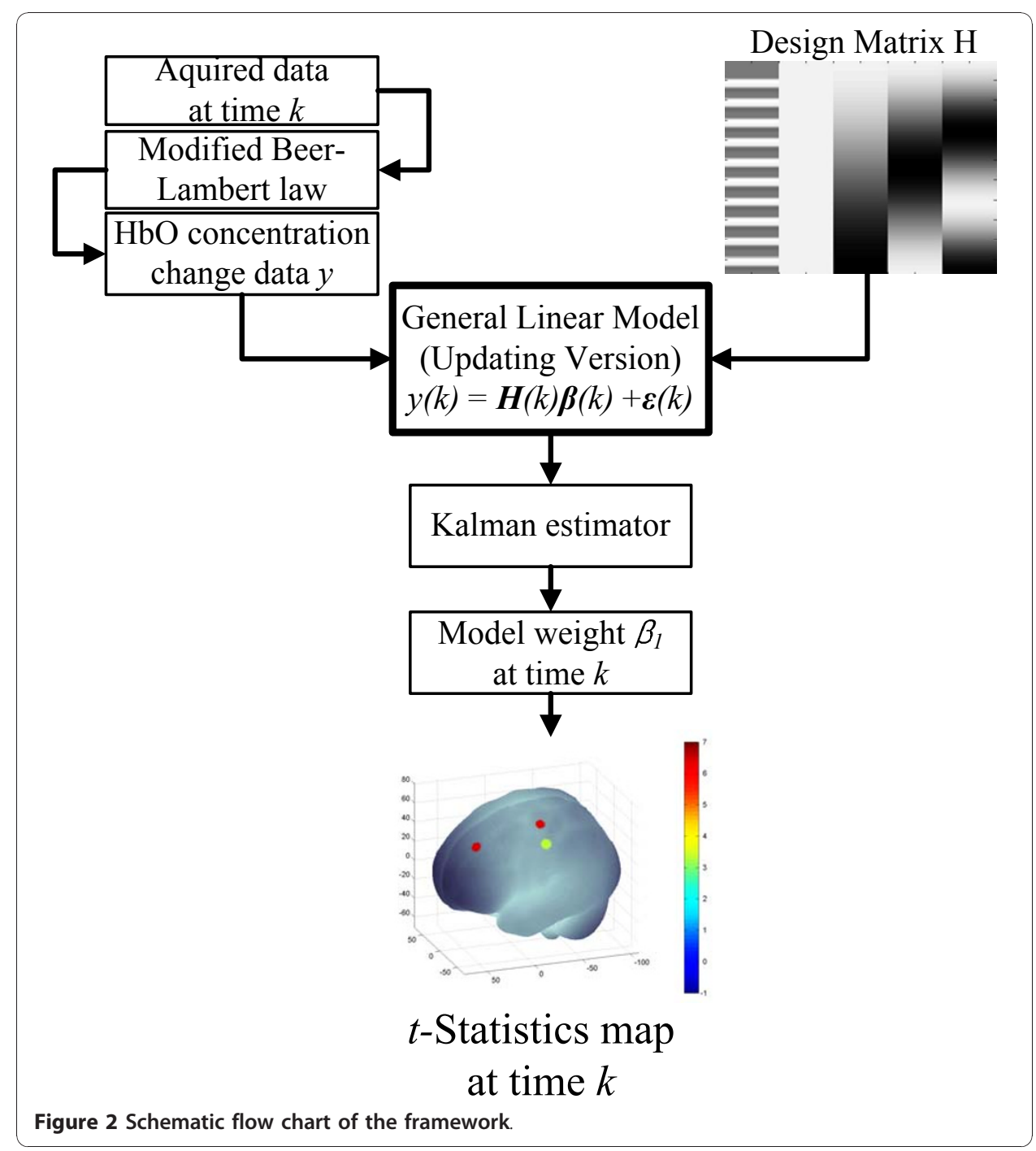

coefficients of the $\mathrm{HbO}$ and $\mathrm{HbR}, L$ is the distance between the source and the detector, and DPF is the differential path length factor. In the present study, the optical density variation was derived by dividing the light intensity measured at each time step by the light intensity measured at the first time step.

\section{General linear model (GLM)}

In NIRS-based studies, both $\mathrm{HbO}$ and $\mathrm{HbR}$ concentration changes can reflect changes in the rCBF. However, it has been suggested that $\mathrm{HbO}$ is a more sensitive indicator of such changes [28]. Therefore, only the $\mathrm{HbO}$ concentration change data was considered in the present study.

The GLM design process is described in detail in [29]; we provide only a brief description here. A design matrix $H$ including a set of explanatory variables is predefined in order to model the observed NIRS time series. Five explanatory variables are considered. The first variable models the $\mathrm{HbO}$ concentration changes (the brain activity signals) using a stimulus vector convolved with the basis function (BF, a doublegamma model; [30]). The second one models the baseline level, and the remaining 
variables represent a set of high-pass filters (discrete cosine transform, DCT) [30] with a cut-off frequency of $0.0006 \mathrm{~Hz}$.

At time $k, y^{i}(k)$, the measured NIRS data of channel $i$, is predicted by $H(k)$, specifically by multiplying a coefficient vector plus an error term $\varepsilon^{i}(k)$. The model can then be expressed as

$$
\gamma^{i}(k)=H(k) \beta^{i}(k)+\varepsilon^{i}(k)
$$

where $\beta^{i}(k)$ is the coefficient vector quantifying the magnitude of the explanatory variables. In vector $\beta^{i}(k)$, we are interested in the component $\beta_{1}^{i}(k)$, which reflects the magnitude of the task-evoked brain response: By statistically determining if it is greater than zero, the existence of brain activity at the area covered by channel $i$ can be confirmed (it is greater than zero) or ruled out (it is less than zero).

Several physiological processes are known to produce temporal correlation in NIRS data, which might lead to inflated $t$-values, and thus underestimation of brain activity. One way to deal with this problem is to make the model fit an AR $(p)$ model (an autoregressive model of the order $p$ ). This leads to a decomposition of the error term $\varepsilon$ into a systematic and a model conform error part. After this, the AR transformation coefficient is applied to both sides of the regression equation

$$
\gamma^{i}(k)-\rho \gamma^{i}(k-1)=H(k)-\rho H(k-1) \beta^{i}(k)+u^{i}(k)
$$

where $\rho$ is the estimated autocorrelation coefficient in an $\mathrm{AR}(1)$ process, and $u(k)=$ $\rho \varepsilon(k-1)+\varepsilon(k)$. By redefining each transformed variable, that is, $y^{i *}(k)=y^{i}(k)-\rho y^{i}(k-1)$, $H^{*}(k)=H(k)-\rho H(k-1)$, one can simplify Equation (3) to

$$
\gamma^{i^{*}}(k)=H^{*}(k) \beta^{i}(k)+u^{i}(k)
$$

It is worth noting that we make an assumption, $|\beta(k)-\beta(k-1)|<\zeta$, for the $\operatorname{AR}(1)$ model used on-line in the current study, where $\zeta>0$ is an arbitrary small number. This assumption compromises the model's robustness. However, as the result shows, the temporal correlation can be effectively reduced. In the present study, $\beta^{i}(k)$ was updated with a Kalman estimator.

\section{Kalman estimator}

The Kalman filtering method is a recursive tracking scheme that estimates the state of a process using an updated regularized linear inversion routine [31]. After decades of development, the Kalman filtering is very mature. Due to its remarkable estimation performance, the Kalman filtering is widely used in many areas [32-34] including neuroscience $[24,25,35,36]$. In the present study, the Kalman filter was used as a model coefficients estimator. The model coefficients from all of the 24 channels were updated in parallel. For a given channel, the state vector, transition equation and observation equation can be described in the form

$$
\begin{aligned}
& X(k)=\left[\begin{array}{llll}
\beta_{1}(k) & \beta_{2}(k) & \ldots & \beta_{L}(k)
\end{array}\right]^{T} \\
& X(k)=A \widehat{X}(k-1)+w(k)
\end{aligned}
$$




$$
y(k)=H(k) X(k)+v(k)
$$

where $L$ is the number of explanatory variables. The state is assumed to follow a random walk with zero drift over time: Thus, $A$ equals the identity matrix, and the process noise $w(k) \sim N(0, Q), y(k)$ is the measured data, $H(k)$ is the vector of explanatory variables, and the observation noise $v(k) \sim N(0, R)$. The filter performs state estimation by the iterative process

$$
\begin{aligned}
& \hat{X}^{-}(k)=A \hat{X}(k-1) \\
& P^{-}(k)=A P(k-1) A^{T}+Q \\
& K(k)=P^{-}(k) H^{T}(k) E^{-1}(k) \\
& \hat{X}(k)=\hat{X}^{-}(k)+K(k) \Delta y(k) \\
& P(k)=(I-K(k) H(k)) P^{-}(k),
\end{aligned}
$$

where $E(k)=H(k) P^{-}(k) H^{T}+R, \Delta y(k)=\gamma(k)-H(k) X^{-}(k), K(k)$ is the Kalman gain, and $P(k)$ is the updated error covariance matrix. In this notation, the superscript (-) refers to the intermediate state and covariance predictions provided by the state update model, which are then modified by the measured data to produce the next state value. In the present study, the state vector was initialized to zero. The a priori estimates of the process and observation noise covariances $\left(Q\right.$ and $R$ respectively) were $(1 \% / \mathrm{sec})^{2}$ and $(0.5 \mu \mathrm{M} / \mathrm{sec})^{2}$, according to a restricted maximum likelihood (ReML) estimation and an empirical-experimental performance check based on a set of training data. We collected the training data during 3 sessions of finger tapping for each subject. We estimated the $Q$ and $R$ values in two steps: They were estimated separately from each of the subjects by ReML, averaged, and then adjusted according to the performance in practically estimation based on the training data.

\section{$t$-Statistics}

The estimated model coefficient vector $\beta$ was used to calculate a relevant $t$-value for a one-tailed $t$-test to test the null hypothesis $c^{T} \beta=0$ [29]. In the present study, the $t$-statistics of channel $i$ at time step $k$ were obtained using

$$
t^{i}(k)=\frac{c^{T} \beta^{i}(k)}{\sqrt{\hat{\sigma}^{i 2}(k) c^{T}\left[\sum_{1}^{k} H_{k}^{T} H_{k}\right]^{-1} c}},
$$

where $c$ is a vector of contrast for selecting the coefficient of interest [29], and $\hat{\sigma}^{i 2}$ is the residual sum-of-squares divided by the appropriate degrees of freedom, and is given by

$$
\hat{\sigma}^{i 2}(k)=\frac{1}{k-L} \sum_{1}^{k}\left[y_{k}^{i}-H_{k} \beta_{k}^{i}\right]^{2},
$$


where $L$ is the number of regressors. Therefore, the null hypothesis $c^{T} \beta_{i}(k)=0$ was assessed by comparing $t^{i}(k)$ with a $t$-distribution with $k-L$ degrees of freedom. By setting proper $p$-values with Bonferroni correction, a statistical activation map of the detected area could be displayed.

\section{Results}

To simulate a real-time process, each measured data was incorporated sequentially in the analysis and updated at each time step. The entire procedure was simulated with Matlab at this stage. The computation time for one processing step was approximately $0.015 \pm 0.0025 \mathrm{sec}$ (mean \pm standard deviation, 4 subjects averaged).

Figure 3 depicts the raw data, and the estimated and $t$-statistics values of two representative channels from subject 1: the activated area (channel 6) and an inactivated area (channel 22). The raw NIRS time series are plotted in the top panels. The time evolutions of the estimated $\beta_{1}$ are plotted in the middle panels. The corresponding $t$-statistics are plotted in the bottom panels. These values were estimated after the 6th sampling.

The motor cortex brain activity related to the finger-tapping task was found in 4 out of the 5 subjects. In the case of the fifth subject, there was no brain activation identified by the proposed framework. Figure 4 shows representative snapshots of the probability activation map ( $t$-statistics map) at the different times $T=120,200,300,400,500$, and 552 sec, from the top row (a) to the bottom row (f) of the detected area, for subjects 1 to 4 . Row (g) shows the activation map estimated by the conventional method (the off-line GLM-based method, ordinary least squares followed by $t$-statistics). The $p$-value set for an individual test was $p_{\text {in }}<0.05$. At an early stage, around $120 \mathrm{sec}$, the proposed method could track the finger-tapping-related brain-active area, as indicated in Table 1 column
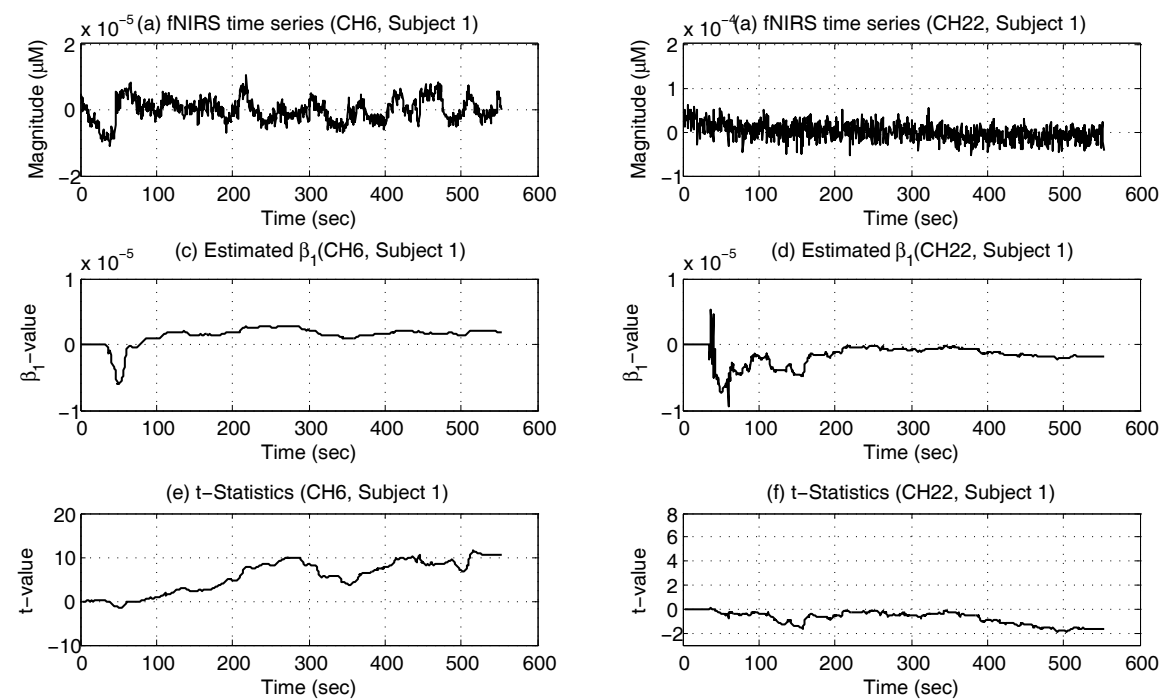

Figure 3 Representative plots of different quantities estimated on-line. Panels (a) and (b) show the NIRS time series measured from the representative channels. Panels (c) and (d) show separately the estimated critical model coefficient $\beta_{1}$. Panels (e) and ( $f$ ) show separately the calculated $t$-statistics. 


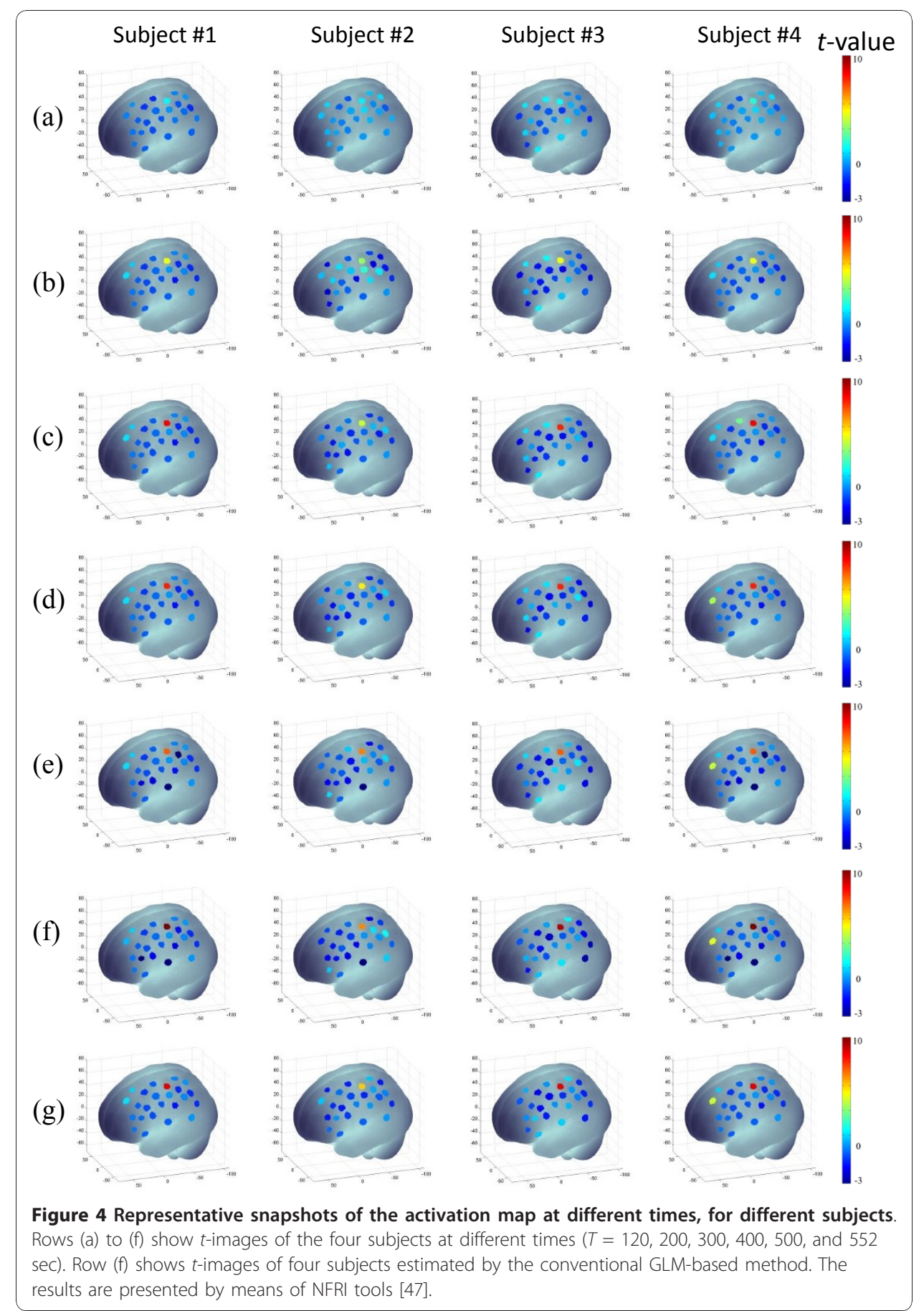

3. With Bonferroni correction, the time for the method to track the brain-active area was delayed by around $60 \mathrm{sec}$, as shown in Table 1 column 4 . The estimated anatomical location of channel 6 was Broadmann area 4 (primary motor cortex), and of channel 4, Broadmann area 8 . The result estimated by our framework was compared with the result estimated by the classical method. We found that at the final stage of the experiment, our results were almost consistent with those estimated by the conventional method. It 
Table 1 Time and location of the brain activities tracked

\begin{tabular}{cccc}
\hline Subject & $\begin{array}{c}\text { Channel } \\
\text { (location) }\end{array}$ & $\begin{array}{c}\text { Time }[\mathbf{s e c}] \\
\left(\boldsymbol{p}_{\text {in }}<\mathbf{0 . 0 5}\right)\end{array}$ & $\begin{array}{c}\text { Time }[\mathbf{s e c}] \\
\left(\boldsymbol{p}_{\boldsymbol{b}}, \text { with Bonferroni correction) }\right)\end{array}$ \\
\hline 1 & 6 & 118 & 177 \\
\hline 2 & 6 & 115 & 170 \\
\hline 3 & 6 & 129 & 186 \\
\hline 4 & 4 & 335 & 402 \\
\hline & 6 & 125 & 184 \\
\hline
\end{tabular}

is noteworthy that our method was able to locate and track the brain-active areas while the experiment was running and, thereby, to provide feedback on-line.

\section{Discussion}

Changes in cerebral oxygenation reflect cerebral functional activity. In the present work, an updated version of the GLM was devised and used for on-line brain activation mapping. To demonstrate this framework, a finger-tapping task activation mapping study was carried out. This study allowed us to highlight several important features of our framework.

An obvious advantage of our method is its real-time applicability. The Kalman estimator has an acceptable computational overload, which allowed us to implement this method using Matlab in real-time $(1.81 \mathrm{~Hz}$ in this study). The proposed method displays an updated brain activation map while the experiment is running. The fingertapping-related brain-active area can be identified and tracked at the early stage of an experiment. The data analysis stage in the classical GLM framework can be conducted only after an experiment, and thus needs extra time. By contrast, with the proposed method, the model coefficients are recursively estimated, and the brain activation map can be updated at each time step. Accordingly, the method is able to track the brainactive area in an ongoing experiment, and provide an early warning to the experimenter when the subject is not responding appropriately or the system is not working properly or well. Thus, both the subject/patient and researcher can receive feedback in real-time.

There are several physiological noises in NIRS data, including noises caused by cardiac and respiratory activity and blood pressure (Mayer wave) fluctuations, which might cause temporal correlation in the form of inflated $t$-values [27]. Ignoring these noises can lead to overestimation of brain activity. In [24,37], sine functions at different frequencies were used to model these noises; resultantly, some nonlinear terms were added to the classical GLM, and an extended Kalman filter was used to update the magnitude and phase shift of the noise terms. In the proposed framework, we used an AR(1) model to reduce the temporal correlation. We compared the effect of temporal correlation reduction using the two methods. Figure 5 indicates that both methods were able to reduce the temporal correlation in the NIRS time series and, moreover, that the AR(1) model performed better.

Finally, the GLM combined with the Kalman filtering allowed for avoidance of verylow-frequency noises in the process of real-time estimation. In NIRS time series, very-low-frequency noises caused by optodes shifts or possible slow cardiac/respiratory artifacts sometimes exist. The upper plot in Figure 6 provides a sample data series containing very-low-frequency noise. The data in this plot was recorded at channel 19, 


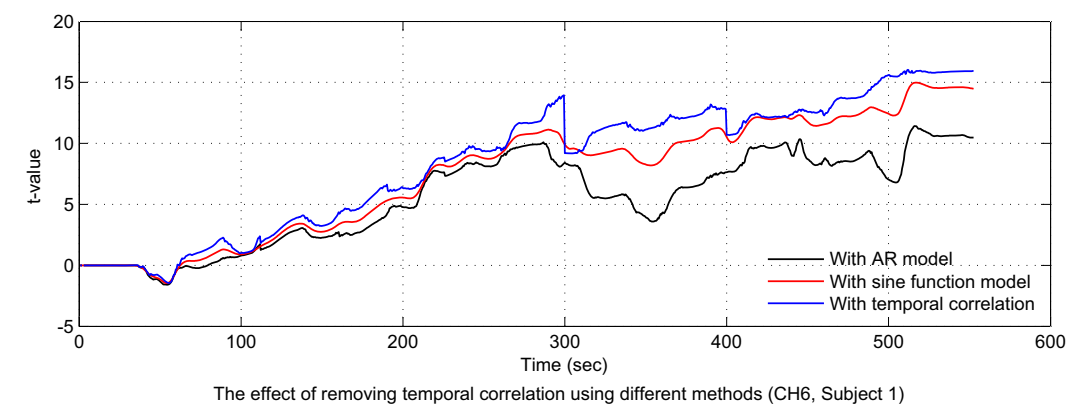

Figure 5 Effect of temporal correlation reduction in NIRS time series. The time course of inflated tvalues caused by temporal correlation is shown in the figure. The effects of two different methods (the sine function model and the AR model) in reducing the temporal correlation are compared.

subject 3. This channel covered the area of Bordemann area 9, where no brain activity was expected to be found in the current experiment. A clear slow shift of the baseline level can be identified by the human eye. In this study, a set of high-pass filters (discrete cosine transform, DCT) was modelled as regressors in the design matrix to remove the very-low-frequency noises on-line. The lower plot in Figure 6 shows estimation results using the DCT filter in comparison with those without using the DCT filter. This makes clear that without using the DCT filter, the estimation results were severely distorted by the low-frequency noise, which therefore would lead to a biased statistical analysis result.

There are some limitations of our method. First, the design matrix in the GLM was designed before the experiment. Different subjects might have individual hemodynamic
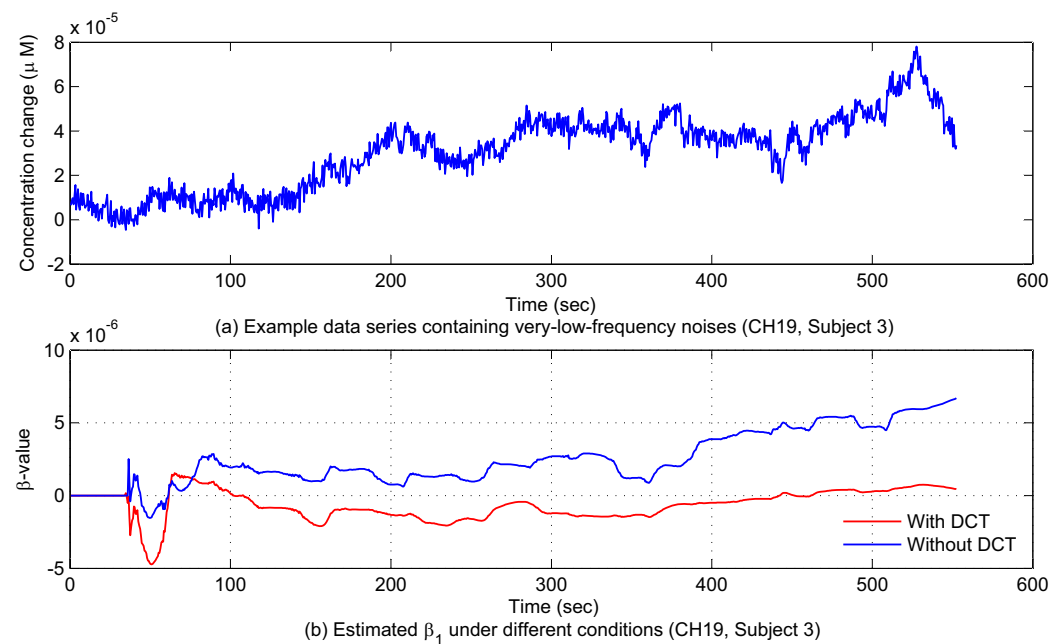

Figure 6 Very-low-frequency noises and the DCT filter effect. Panel (a) gives an example of a piece of NIRS data containing very-low frequency noises. Panel (b) shows the DCT filter preventing very-low frequency noises from leading to biased results. 
responses. However, we assumed a general hemodynamic response model for different subjects in our framework. Our method might not work properly if a notable difference exists between the hemodynamic response of a subject and our modelled one. In [38], a set of linear combined Gaussian-based temporal functions was used to model the hemodynamic response. This model can approximate complex hemodynamic responses among different subjects, by means of multiple overlapping Gaussian functions. However, a recent work [39] suggested that event-evoked hemodynamic responses were similar among different subjects. In the current study, we also found a similarity in the hemodynamic responses, among all of our subjects. Therefore, we consider that it was sufficient to use the general hemodynamic model for estimation in current study.

The second limitation lies in the fact that we made only a preliminary conclusion about the feasibility of the proposed on-line framework, based on the results from 5 subjects. In the current study, brain activity at the primary motor cortex was found in 4 out of the 5 subjects investigated using the proposed framework. There was no brain activation area identified for subject 5 , because the measurement noises (electric voltage fluctuations) induced by the equipment were very large in the case of this subject. Thus, no brain-active area could be identified based on the measured data. In order to produce a complete picture of the feasibility of the proposed framework, data needs to be measured for a greater number of subjects.

The $t$-statistics from the current study are based on the estimation of mean square error (MSE) via Equation (14). Therefore, while the experiment is running, the cumulative type I error increases as the test number grows. Thus, a post-hoc analysis is also needed to guarantee the statistical significance. We used Bonferroni correction for posthoc analysis at each step in the current study. The $p_{\text {in }}$ for an individual test was set to 0.05 , and the $p_{b}$ after Bonferroni correction was calculated as $p_{b}=\left(1-\left(1-p_{i n}\right)^{j}\right] / j$, where $j$ represents the number of tests. As indicated in Table 1 column 4, the post-hoc analysis reduced the performance of the proposed framework, though it still yields promising results. Nevertheless, it might not be suitable for long-duration experiments (e.g. 6 hours' measurement for sleep studies). When the number of tests increases to a large value, the $p_{b}$ might be difficult to satisfy, resulting in an underestimation of brain activation. This is one of the limitations of the proposed framework. The problem probably can be avoided, either by reducing the sampling frequency over a long-duration measurement, thereby reducing the number of tests, or by controlling the experimentation time.

The time needed for locating different brain-active areas differed in the current study. Because Kalman filtering is a recursive process, the new information will be added as it arrives at each sampling time. Therefore, the estimation results from early stages are less reliable than those from later ones. In another aspect, the brain is a heterogeneous and dynamic organ that can process parallel work (in different brain areas), and so the same estimation process might perform differently for different brain areas. In our study, channel 4 of subject 4 was identified as a brain-active area during the finger-tapping task. The area covered by channel 4 is Brodmann area 8 , responsible for voluntary eye movement, which is not expected to be active during finger tapping. This particular result might be accounted for simply as an indication that subject 4 was moving his eyes frequently while tapping his finger. 
The DYNOT equipment in the present study employs a brush-less DC servomotor to provide for light source beam positioning [40]. The motor is driven by a controller containing freely programmable microprocessor. In this experiment, only a simple open-loop control scheme was designed for controlling the motor moving. Some complex control algorithms can be applied to achieve a more precise motor controlling [41-46].

One further issue in NIRS topographical applications is the fact that only a small portion of a detected area is measurable with sparsely distributed channels. Either tomographical NIRS detection or application of interpolation techniques might help increase it [20]. Both solutions would require extra processing time. Therefore, the issue of the development of a proper means of achieving higher spatial resolution in real-time applications also will have to be resolved in future research.

\section{Conclusions}

A new Kalman-estimator- and GLM-based NIRS data analysis framework was demonstrated for real-time imaging of brain activity. In an experiment, this framework allowed for updating of the topographic activation map of the detected brain area, and, additionally, it could locate the activated brain area at an early stage by analyzing the noise-containing raw data.

\section{Acknowledgements}

This research was supported by the World Class University program through the National Research Foundation of Korea funded by the Ministry of Education, Science and Technology, Korea (grant no. R31-2008-000-20004-0).

\section{Author details}

${ }^{1}$ Department of Cogno-Mechatronics Engineering, Pusan National University; 30 Jangjeon-dong, Geumjeong-gu, Busan609-735, Korea. 'Department of Cogno-Mechatronics Engineering and School of Mechanical Engineering, Pusan National University; 30 Jangjeon-dong, Geumjeong-gu, Busan 609-735, Korea. ${ }^{3}$ Department of Electrical and Computer Engineering, The National University of Singapore, Singapore 117576.

\section{Authors' contributions}

XSH performed the experiment and carried out the data processing. KSH participated in the data processing and revised the manuscript. SSG participated in theoretical aspects of the study design and evaluated the experiment. MYZ evaluated the experiments and gave suggestions to the manuscript. All of the authors read and approved the final manuscript.

\section{Competing interests}

The authors declare that they have no competing interests.

Received: 5 July 2010 Accepted: 8 December 2010 Published: 8 December 2010

\section{References}

1. Boas DA, Dale AM, Franceschini MA: Diffuse optical imaging of brain activation: approaches to optimizing image sensitivity, resolution, and accuracy. Neuroimage 2004, 23:S275-S288.

2. Koizumi H, Yamamoto T, Maki A, Yamashita Y, Sato H, Kawaguchi H, Ichikawa N: Optical topography: practical problems and new applications. Appl Opt 2003, 42:3054-3062.

3. Obrig H, Villringer A: Beyond the visible-imaging the human brain with light. J Cereb Blood Flow Metab 2003, 23:1-18.

4. Wolf $M$, Ferrari $M$, Quaresima V: Progress of near-infrared spectroscopy and topography for brain and muscle clinical applications. J Biomed Opt 2007, 12:062104.

5. Perrey S: Non-invasive NIR spectroscopy of human brain function during exercise. Methods 2008, 45:289-299.

6. Toronov $\mathrm{V}$, Zhang $X$, Webb AG: A spatial and temporal comparison of hemodynamic signals measured using optical and functional magnetic resonance imaging during activation in the human primary visual cortex. Neuroimage 2007, 34:1136-1148.

7. Barbour RL, Graber HL, Pei YL, Zhong S, Schmitz CH: Optical tomographic imaging of dynamic features of densescattering media. Journal of the Optical Society of America a-Optics Image Science and Vision 2001, 18:3018-3036.

8. Irani F, Platek SM, Bunce S, Ruocco AC, Chute D: Functional near infrared spectroscopy (fNIRS): An emerging neuroimaging technology with important applications for the study of brain disorders. Clinical Neuropsychologist 2007, 21:9-37. 
9. Gratton G, Fabiani M: The event-related optical signal: a new tool for studying brain function. International Journal of Psychophysiology 2001, 42:109-121.

10. Cope M, Delpy DT, Reynolds EO, Wray S, Wyatt J, van der Zee P: Methods of quantitating cerebral near infrared spectroscopy data. Adv Exp Med Biol 1988, 222:183-189.

11. Zhao H, Tanikawa Y, Gao F, Onodera Y, Sassaroli A, Tanaka K, Yamada Y: Maps of optical differential pathlength factor of human adult forehead, somatosensory motor and occipital regions at multi-wavelengths in NIR. Phys Med Biol 2002, 47:2075-2093.

12. Hashimoto T, Minagawa-Kawai Y, Kojima S: Motion illusion activates the visual motion area of the brain: A nearinfrared spectroscopy (NIRS) study. Brain Research 2006, 1077:116-122.

13. Shibuya K, Kuboyama N: Human motor cortex oxygenation during exhaustive pinching task. Brain Research 2007, 1156:120-124.

14. Watanabe H, Homae F, Nakano T, Taga G: Functional activation in diverse regions of the developing brain of human infants. Neuroimage 2008, 43:346-357.

15. Huppert TJ, Diamond SG, Franceschini MA, Boas DA: HomER: a review of time-series analysis methods for nearinfrared spectroscopy of the brain. Applied Optics 2009, 48:D280-D298.

16. Schroeter ML, Bucheler MM, Muller K, Uludag K, Obrig H, Lohmann G, Tittgemeyer M, Villringer A, von Cramon DY: Towards a standard analysis for functional near-infrared imaging. Neuroimage 2004, 21:283-290.

17. Koh PH, Glaser DE, Flandin G, Kiebel S, Butterworth B, Maki A, Delpy DT, Elwell CE: Functional optical signal analysis: a software tool for near-infrared spectroscopy data processing incorporating statistical parametric mapping. J Biomed Opt 2007, 12:064010.

18. Lu CM, Zhang YJ, Biswal BB, Zang YF, Peng DL, Zhu CZ: Use of fNIRS to assess resting state functional connectivity. J Neurosci Methods 186:242-249.

19. Plichta MM, Heinzel S, Ehlis AC, Pauli P, Fallgatter AJ: Model-based analysis of rapid event-related functional nearinfrared spectroscopy (NIRS) data: a parametric validation study. Neuroimage 2007, 35:625-634.

20. Ye JC, Tak S, Jang KE, Jung J, Jang J: NIRS-SPM: statistical parametric mapping for near-infrared spectroscopy. Neuroimage 2009, 44:428-447.

21. Worsley KJ, Friston KJ: Analysis of fMRI time-series revisited-again. Neuroimage 1995, 2:173-181.

22. McGilchrist CA, Sandland RL: Recursive Estimation of the General Linear Model with Dependent Errors. Journal of the Royal Statistical Society 1979, 41:65-68.

23. Bagarinao E, Matsuo K, Nakai T, Sato S: Estimation of general linear model coefficients for real-time application. Neuroimage 2003, 19:422-429.

24. Abdelnour AF, Huppert T: Real-time imaging of human brain function by near-infrared spectroscopy using an adaptive general linear model. Neuroimage 2009, 46:133-143.

25. Buchel C, Friston KJ: Dynamic changes in effective connectivity characterized by variable parameter regression and Kalman filtering. Hum Brain Mapp 1998, 6:403-408.

26. Obrig H, Neufang M, Wenzel R, Kohl M, Steinbrink J, Einhaupl K, Villringer A: Spontaneous low frequency oscillations of cerebral hemodynamics and metabolism in human adults. Neuroimage 2000, 12:623-639.

27. Matthews F, Pearlmutter BA, Ward TE, Soraghan C, Markham C: Hemodynamics for braincomputer interfaces. IEEE Signal Processing Magazine 2008, 25:87-94.

28. Hoshi Y, Kobayashi N, Tamura M: Interpretation of near-infrared spectroscopy signals: a study with a newly developed perfused rat brain model. J Appl Physiol 2001, 90:1657-1662.

29. Friston KJ: Statistical parametric mapping: the analysis of funtional brain images. 1 edition. Amsterdam; Boston: Elsevier/ Academic Press; 2007.

30. Glover GH: Deconvolution of impulse response in event-related BOLD fMRI. Neuroimage 1999, 9:416-429.

31. Kalman RE: A new approach to linear filtering and prediction problems. Journal of Basic Engineering 1960, 82:35-45.

32. Kim YS, Hong KS: An IMM algorithm with federated information mode-matched filters for AGV. International Journal of Adaptive Control and Signal Processing 2007, 21:533-555.

33. Kim YS, Hong KS: Federated information mode-matched filters in ACC environment. International Journal of Control Automation and Systems 2005, 3:173-182.

34. Turnip A, Hong KS, Park S: Modeling of a hydraulic engine mount for active pneumatic engine vibration control using the extended Kalman filter. Journal of Mechanical Science and Technology 2009, 23:229-236.

35. Diamond SG, Huppert TJ, Kolehmainen V, Franceschini MA, Kaipio JP, Arridge SR, Boas DA: Physiological system identification with the Kalman filter in diffuse optical tomography. Medical Image Computing and Computer-Assisted Intervention - Miccai 2005, Pt 2 2005, 3750:649-656.

36. Izzetoglu M, Chitrapu P, Bunce S, Onaral B: Motion artifact cancellation in NIR spectroscopy using discrete Kalman filtering. Biomed Eng Online 9:16.

37. Prince S, Kolehmainen V, Kaipio JP, Franceschini MA, Boas D, Arridge SR: Time-series estimation of biological factors in optical diffusion tomography. Physics in Medicine and Biology 2003, 48:1491-1504.

38. Diamond SG, Huppert TJ, Kolehmainen V, Franceschini MA, Kaipio JP, Arridge SR, Boas DA: Dynamic physiological modeling for functional diffuse optical tomography. Neuroimage 2006, 30:88-101.

39. Huppert TJ, Hoge RD, Diamond SG, Franceschini MA, Boas DA: A temporal comparison of BOLD, ASL, and NIRS hemodynamic responses to motor stimuli in adult humans. Neuroimage 2006, 29:368-382.

40. Schmitz CH, Locker M, Lasker JM, Hielscher AH, Barbour RL: Instrumentation for fast functional optical tomography. Review of Scientific Instruments 2002, 73:429-439.

41. Bentsman J, Hong KS: Vibrational Stabilization of Nonlinear Parabolic-Systems with Neumann Boundary-Conditions. IEEE Transactions on Automatic Control 1991, 36:501-507.

42. Bentsman J, Hong KS, Fakhfakh J: Vibrational Control of Nonlinear Time-Lag Systems - Vibrational Stabilization and Transient-Behavior. Automatica 1991, 27:491-500.

43. Hong KS: An open-loop control for underactuated manipulators using oscillatory inputs: Steering capability of an unactuated joint. IEEE Transactions on Control Systems Technology 2002, 10:469-480.

44. Sohn HC, Hong KT, Hong KS, Yoo WS: An adaptive LQG control for semi-active suspension systems. International Journal of Vehicle Design 2004, 34:309-326. 
45. Choi JY, Hong KS, Yang KJ: Exponential stabilization of an axially moving tensioned strip by passive damping and boundary control. Journal of Vibration and Control 2004, 10:661-682.

46. Yang KJ, Hong KS, Matsuno F: Energy-based control of axially translating beams: Varying tension, varying speed, and disturbance adaptation. IEEE Transactions on Control Systems Technology 2005, 13:1045-1054.

47. Singh AK, Okamoto M, Dan H, Jurcak V, Dan I: Spatial registration of multichannel multi-subject fNIRS data to MNI space without MRI. Neuroimage 2005, 27:842-851.

doi:10.1186/1475-925X-9-82

Cite this article as: Hu et al:: Kalman estimator- and general linear model-based on-line brain activation mapping by near-infrared spectroscopy. BioMedical Engineering OnLine 2010 9:82.

Submit your next manuscript to BioMed Central and take full advantage of:

- Convenient online submission

- Thorough peer review

- No space constraints or color figure charges

- Immediate publication on acceptance

- Inclusion in PubMed, CAS, Scopus and Google Scholar

- Research which is freely available for redistribution 